\title{
An interdisciplinary postdoctoral fellowship model: Opportunities for nurse PhDs
}

\author{
Michaela S. McCarthy*1,2, Jessica-Jean Stonecipher ${ }^{1,3}$, Heather Gilmartin ${ }^{1,4}$, Dana El-Hajj ${ }^{1,2}$, Catherine Battaglia ${ }^{1,4}$ \\ ${ }^{1}$ Veterans Health Administration, Eastern Colorado Health Care System, Denver-Seattle Center of Innovation, Aurora, Colorado, \\ USA \\ ${ }^{2}$ University of Colorado, College of Nursing, Aurora, Colorado, USA \\ ${ }^{3}$ University of Florida, University Writing Program, Gainesville, Florida, USA \\ ${ }^{4}$ University of Colorado, School of Public Health, Aurora, Colorado, USA
}

Received: July 31, 2019

DOI: $10.5430 /$ jnep.v10n2p33
Accepted: October 8, 2019

Online Published: November 5, 2019

\begin{abstract}
Interdisciplinary postdoctoral fellowships can provide rich opportunities for nurses to receive additional training and develop diverse professional academic and research partnerships. They provide a structure for learning in which team science is emphasized and complex health issues are addressed. This paper presents an interdisciplinary postdoctoral fellowship model and highlights the development of one nurse fellow's network during the program. The fellowship curriculum is outlined and the three focus areas (education, research, and experience) are further explained. A social network analysis approach was used to illustrate the growth in one nurse fellow's network during a two-year postdoctoral fellowship. The first year of the fellowship showed an increase in the number of professional connections, while in the second year the relationships deepened as collaborations were established and strengthened.
\end{abstract}

Key Words: Nursing, Postdoctoral fellowship, Interdisciplinary

\section{INTRODUCTION}

The challenges facing healthcare today are complex, multifactorial, and require problem-driven research partnerships tailored to address scientific and societal challenges in innovative ways. ${ }^{[1]}$ Interdisciplinary research programs can be one approach to solve the complex healthcare problems of the 21 st century. ${ }^{[2]}$ Interdisciplinary research is defined as a mode of research by teams or individuals that integrates information, data, techniques, tools, perspectives, concepts, and/or theories from two or more disciplines to advance fundamental understanding or to solve problems whose solutions are beyond the scope of a single discipline or area of research practice. ${ }^{[1]}$ In healthcare, interdisciplinary teams include different healthcare providers as well as professionals from other disciplines such as science, anthropology, and economics. Successful interdisciplinary investigators find ways to integrate and synthesize the depth of their professional and educational knowledge with those of disparate backgrounds to explore problems and questions that are outside the reach of any single discipline. For example, interdisciplinary research in genomics and bioinformatics have resulted in large scale collaborations and discoveries, like the Human Genome Project, that are changing the landscape of science. ${ }^{[1]}$

Despite the promises of interdisciplinary research, investi-

\footnotetext{
*Correspondence: Michaela S. McCarthy; Email: Michaela.mccarthy@va.gov; Address: Denver-Seattle Center of Innovation, 1700 N. Wheeling, Bldg P1-151, Aurora, Colorado, 80045, USA.
} 
gators often face obstacles achieving necessary partnerships. These include cultural and language barriers between fields that silo investigators into specialty areas, where efforts remain disconnected from the greater whole. ${ }^{[1]}$ Another obstacle is the tradition of organizing research activities by discipline-based departments. This occurs in academic institutions, funding organizations, professional societies, and journals. $^{[1]}$ To counter increased specialization in science and health, interdisciplinary postdoctoral training programs are being developed to cultivate the next generation of interdisciplinary scientists.

Interdisciplinary postdoctoral training programs support new investigators to build on the research skills developed in graduate school and ensure the individual is adequately equipped to carry out independent research, seek funding, and disseminate findings. ${ }^{[3,4]}$ These programs are focused on interdisciplinary communication, team science, and the creation of a broad network of mentors and collaborators. ${ }^{[1]}$ This model of postdoctoral training is meant to change the academic research culture so that interdisciplinary approaches and team science are increasingly used to conduct research. Moreover, new investigators trained in one discipline are given the opportunity to learn from other disciplines. In the setting of embedded research programs, as in the Veterans Health Administration (VHA), interdisciplinary postdoctoral programs have the potential to translate research into practice more quickly and successfully, by integrating training into new interdisciplinary approaches.

Doctor of Philosophy $(\mathrm{PhD})$ prepared nurse investigators play an important role in the healthcare system. The nursing science community is uniquely positioned to address the healthcare needs of individuals and populations. ${ }^{[5]}$ The American Academy of Nursing has developed a research agenda to inform clinical practice, promote health, and improve patient outcomes. ${ }^{[6]}$ Postdoctoral training is a critical foundation for a successful nursing research career and offers new nurse investigators the opportunity to develop their research agenda, grow their publication portfolio, and further develop research skills to transition into independent investigators. ${ }^{[4]}$ Fellowship training prepares nurse researchers to be independent principal investigators who collaborate with interdisciplinary investigators to understand and address complex health issues. ${ }^{[7]} \mathrm{In}$ an interdisciplinary fellowship, nurse fellows are expected to seek mentorship from senior investigators in multiple disciplines, which creates a robust network of collaborators who can serve as research partners throughout a career. This can serve to expand the nurse fellow's worldview and introduce the nurse fellow to diverse research methodologies. The purpose of this paper is twofold. We will initially describe the underlying conceptual model and curriculum focus areas of an interdisciplinary postdoctoral fellowship. We will subsequently describe how this fellowship design contributed to the social network continuum of a nurse fellow using a social network analysis approach.

\section{VETERANS ADMINISTRATION POSTDOC- TORAL FELLOWSHIP PROGRAM}

The VHA, through the Office of Academic Affiliations (OAA), has a long history of supporting new $\mathrm{PhD}$ and Doctor of Medicine (MD) researchers through postdoctoral fellowship programs in clinical, biological, and health systems research. The goal of the fellowship is to train early stage investigators to impact and improve Veterans' health and to grow the next generation of VHA researchers. Fellowships are designed to support adult learners and are structured to include trainings and interdisciplinary workgroups to encourage collaboration. Multiple aspects of practice, research, and scholarship are targeted to support growth in early stage researchers. In the Eastern Colorado Health Care System (ECHCS), the health services research postdoctoral training program is housed within the Denver-Seattle Center of Innovation (COIN). The COIN's mission includes high-quality research, clinical dissemination of results to improve Veteran care, and to train future health services investigators. Nurses have been trained through both the VHA Postdoctoral Nurse Fellowship and the Advanced Health Services Research \& Development Fellowship. They have advanced their research in conjunction with peers from medicine, psychology, nutrition, medical anthropology, public health, and health services research.

\subsection{Denver postdoctoral fellowship conceptual frame- work}

The Denver based fellowship program was based on a conceptual model of academic persistence developed by Tinto ${ }^{[8]}$ and adapted by Manson ${ }^{[9]}$ (see Figure 1). This model incorporates both fixed and dynamic aspects of postdoctoral fellowships that can lead to academic persistence and success. The fixed aspects of the model incorporate the background and environment of the fellowship, including characteristics of both the trainees and the research environment in which the fellowship is housed. Dynamic aspects of the model include trainee processes throughout the fellowship including social/ecological, cognitive, and use of services. The model includes short-term outcomes such as goals, commitments and academic/scientific development which are pursued during the fellowship. Long-term outcomes such as academic persistence continue to develop throughout the fellows' careers. 


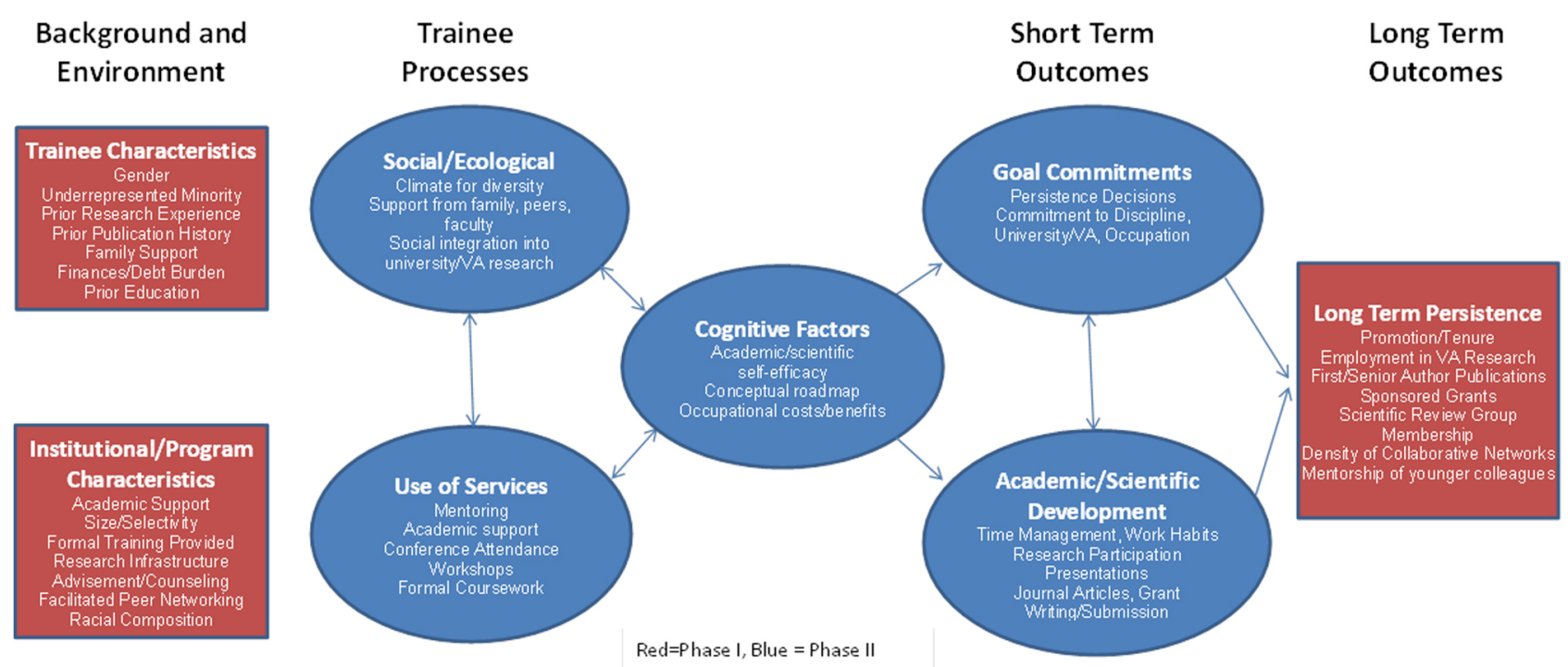

Figure 1. Conceptual model of academic persistence ${ }^{[8,9]}$

\subsection{Fellowship curriculum guidance}

The Denver interdisciplinary postdoctoral fellowship program focuses on three specific areas to support a fellow's development: education, research, and experience (see Figure 2). The education focus area is designed to provide opportunities to train in complex research methodologies necessary for a successful career in health services research. Examples include structural equation modeling, dissemination and implementation strategies, and qualitative methodologies. Additionally, structured protocol and grant writing workshops provide opportunities to strengthen skills necessary for future success.

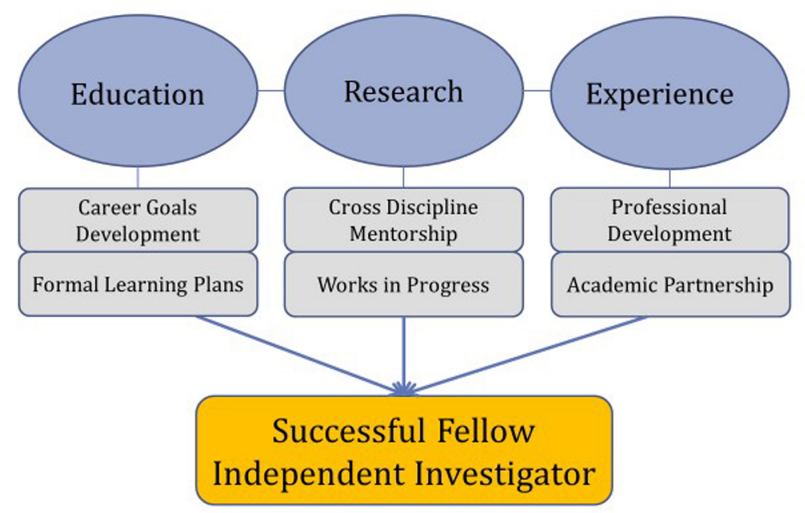

Figure 2. Fellowship focus areas

The goal of the research focus area is to help fellows explore and define effective and novel programs of research to improve Veteran health and/or health care delivery systems improving patient centered care, population health and value. ${ }^{[10]}$ At the end of the fellowship, fellows are ideally well positioned to write an early career grant to continue their research progress.

The experience focus area is designed to encourage fellows to build a network of connections to facilitate both professional and research interests. Each fellow is encouraged to hold an academic appointment and to network with other postdoctoral fellows, academics, and researchers on the affiliated academic campus. Further networking with senior researchers and organizations both nationally and internationally is encouraged.

\subsection{Curriculum components}

To facilitate learning, structured expectations were developed for each area of focus. As a part of the education focus area, fellows create career development plans and formal learning goals. These living documents are updated monthly, and used to review progress and strategize future career development and learning opportunities during quarterly meetings with mentors. The Denver-based fellowship is located on an academic medical campus and fellows are encouraged to take advantage of multiple opportunities for learning across disciplines. Didactic courses are offered through associated health care colleges (medicine, nursing, pharmacy, public health) and multiple organizations across campus have structured seminars and workshops. Fellows are encouraged to attend classes, seminars, and workshops to facilitate networking and learning across disciplines.

In acknowledgement that research, dissemination, and implementation are most effectively conducted with an interdisciplinary team approach, this fellowship is structured to include opportunities for fellows to seek mentorship from experts across disciplines. A primary mentor from the Denver COIN with similar research interests is typically matched 
with each fellow at the beginning of the fellowship. This provides an ongoing relationship with a senior researcher who can provide mentorship at all stages of training. Weekly Works In Progress (WIP) meetings are held with the fellows and the fellowship directors, a nurse scientist and a medical practitioner and investigator. Fellows are guided through increasingly complex aspects of research that include: effective review of the literature, study design, recruitment, management of safety and ethical issues, statistical analysis and interpretation, and scientific writing. Weekly WIP meetings also provide an opportunity for fellows to engage in peer mentoring, which has been shown to improve engagement and productivity of junior investigators. ${ }^{[11]}$

The experience focus area is designed to foster professional development and provide fellows with an opportunity to engage with academic partners and develop competency as a teacher. Fellows are expected to present research findings at national or international conferences annually, which fosters presentation skills and encourages networking opportunities. Additionally, fellows are encouraged to engage with health care students in the classroom, providing lectures across disciplines and teaching courses in affiliated colleges. Engagement with community stakeholders to provide context for dissemination and implementation of research is also encouraged.

\section{NURSING CASE STUDY}

In September 2014, the Denver COIN accepted their first nurse postdoctoral fellow. The following year the Denver COIN accepted additional fellows and formed an interdisciplinary postdoctoral training program. We used a social network analysis approach to document the nurse's professional network growth during the two year fellowship program. Social network analysis (SNA) is a research method designed to study relationships between network actors. ${ }^{[12]}$ It is frequently used to study growth and collaboration in professional networks ${ }^{[13-15]}$ and it can easily characterize the number and strength of connections in an individual's network. This case study used a social network analysis approach to collect, analyze, and visualize data on the professional connections (network actors) a nurse postdoctoral fellow made during the fellowship. This approach allowed us to measure and visualize how the fellow's professional network changed over two years with attention to the size of the network, the characteristics of the network actors, and the types of ties (relationships) present in the network.

In 2018, a personal network survey was used to collect data from the first nurse fellow about her academic and professional collaborators and in what years those collaborations began. Although the fellow had many professional relation- ships such as colleagues in her $\mathrm{PhD}$ and postdoctoral program, data was only gathered on the relationships with people she had 1) co-authored a paper, 2) had a formal mentormentee relationship, or 3) collaborated on research protocols or grants. These data were sorted into three periods reflective of the fellow's progress in the fellowship: pre-fellowship, fellowship year one, and fellowship year two.

The pre-fellowship phase included the professional relationships she had established throughout her 24-year nursing career and 4-year doctoral studies program. Fellowship collaborators included the relationships she established within her first year of the fellowship (September 2015 to August 2016) and the second year of the fellowship (September 2016 to August 2017). No data were collected about professional relationships after the fellowship because this analysis focused solely on growth during the fellowship.

Although distinct from a typical network graph, Figure 3 illustrates the growth and change in the fellows' professional network over the course of the fellowship. The shapes in Figure 3 each represent one professional with whom the fellow collaborated, and they are placed in the concentric ring (phase) in which the relationship originated. For example, in the pre-fellowship phase the fellow met and collaborated with $4 \mathrm{MDs}, 2 \mathrm{PhDs}$ (not RNs), and $8 \mathrm{PhD} / \mathrm{RNs}$. Some of these relationships continued throughout the fellowship. The size of each shape illustrates the number of independent collaborations undertaken with each person. These collaborations include joint presentations, publications and grant applications. Finally, Figure 3 is divided into three sections: University of Colorado, Veterans Administration, and Other Affiliations. This is done to illustrate when relationships were formed outside of an academic or institutional network. Emphasizing the number of collaborators, their professional affiliations, and their professional training helps to illustrate the increased diversity of one fellow's professional networks after a postdoctoral nursing fellowship.

The pre-fellowship phase was comprised primarily of nursing-based professional relationships formed during clinical practice and the $\mathrm{PhD}$ process. Individuals in this phase represented professional collaborations and the intense networking necessary to form a successful $\mathrm{PhD}$ community. In the first year of the fellowship, relationships with many past clinical collaborators receded, and a network of new associates more precisely aligned with the fellow's research interests was established. In the second year of the fellowship the strength of key relationships intensified as more publications and grants were written. Accordingly, fewer new collaborators were introduced in year two.

New relationship growth slowed in the second year. This 
fellow's network shows she was pursuing relationships created within the first year in more meaningful ways during year two. During year two she actively worked on three research protocols and in the year following the fellowship she transitioned into leading an interdisciplinary team in a full-time position with the COIN. Her network connections in year one included MDs, statisticians, a psychologist, and one doctoral nurse. In year two, additional MDs and statisticians were added to her network. Connections represented collaborators from both inside the VA and from new unaffiliated academic institutions. Her relationships continued to develop and deepen as she broadened her interdisciplinary network of collaborators to grow her research.

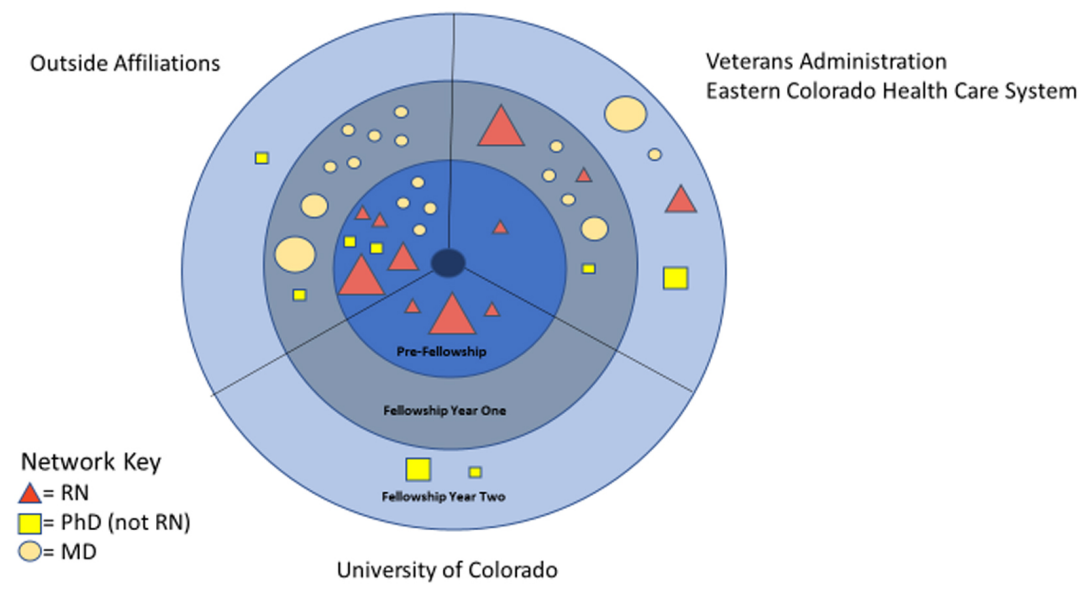

Figure 3. Postdoctoral fellow network

\section{Discussion}

Results of this case study show the professional network growth of one postdoctoral nurse fellow. In the prefellowship phase, the network contained many professional associations primarily within nursing. The large number of pre-fellowship collaborators reflected extensive networking during graduate school as the nurse explored possible research opportunities. As the fellowship began, multiple clinical and professional connections gave way to a more focused and interdisciplinary team approach as the fellow's research program evolved. Partnerships with MDs, statisticians, and other PhD level scientists evolved and strengthened throughout her second year. Many of these new collaborations occurred within the VA where the fellowship was housed, although an almost equal number of collaborations came from outside the VA network (see Figure 3). Products of this work included multiple presentations, publications, and grant applications. This supports the premise that an interdisciplinary postdoctoral fellowship can support a nurse fellow in research growth. The addition of collaborators from multiple disciplines and institutions can further add to professional and research development. The interdisciplinary fellowship provided a strong foundation for research partnerships both internal and external to the home institution as evidenced by the diversity of the fellow's network.

The fellow incorporated the three focus areas of the fellowship (education, research, experience) to grow her interdisci- plinary team. She created an evolving formal learning plan and pursued educational opportunities through the Office of Nursing Services. Career development goals were mapped in conjunction with faculty mentors. She partnered with specialty medical teams to conduct pilot research in her area of interest. Weekly multidisciplinary WIP meetings provided a forum for workshopping articles, presentations and grant proposals. She formed an academic partnership with a school of public health to gain professional experience in coursework development. Additionally she presented at multiple conferences over the course of her fellowship which contributed to the growth of her professional network of collaborators. Each focus area was pursued throughout her fellowship.

\section{Conclusion}

Interdisciplinary postdoctoral fellowships are one way to invest in systematic training of fellows, both through didactic coursework and experiential research. Interdisciplinary collaboration in healthcare is a necessity, and scientists in healthcare are increasingly reliant on interdisciplinary teams to create solutions to complex problems. An interdisciplinary postdoctoral training program is an ideal model for providing opportunities for professionals from different disciplines to work together to create innovative and effective research programs.

The case study presented was that of an experienced family nurse practitioner who had recently graduated with a $\mathrm{PhD}$ in 
nursing. It is likely that her clinical experience contributed to her success in networking. While each postdoctoral fellow enters the program with differing experience, the pursuit of the fellowship focus areas and the tailored mentoring provides a structured path to transition each fellow from a clinical/academic background into a research career.

The evolution of an interdisciplinary postdoctoral fellowship model could evolve beyond just the inclusion of healthcare providers to include participants from other disciplines such as informatics, economics, and user centered design. This approach will provide the opportunity for fellows to gain a broader understanding of a myriad of strategies to solve complex healthcare challenges. A structured postdoctoral program that incorporates education, research, and experience offers the opportunity for new MDs and PhDs to publish and craft their nascent research programs with the guidance of experts from multiple fields.

\section{ACKNOWLEDGEMENTS}

The views and opinions expressed in this article are those of the authors and do not necessarily reflect the official policy or position of any agency of the U.S. government, agency of the State of Colorado, academic institution, or the institutions and organizations with which the authors are affiliated.

\section{CONFlicts OF INTEREST Disclosure}

The authors declare that there is no conflict of interest.

\section{REFERENCES}

[1] Andreasen NC, Brown TL. Facilitating interdisciplinary research. Washington, D.C.: The National Academies Press; 2005.

[2] Scaffidi AK, Berman JE. A positive postdoctoral experience is related to quality supervision and career mentoring, collaborations, networking and a nurturing research environment. Higher Education. 2011; 62(6): 685-698. https ://doi.org/10.1007/s10734-011 $-9407-1$

[3] Montpetit AJ. A kangaroo and your research toolbox. Biological Research for Nursing. 2011; 13(4): 337-339. PMid:22058074 https://doi.org/10.1177/1099800411413915

[4] National Postdoctoral Association (NPA). The NPA postdoctoral core competencies toolkit [Internet]. 2010 - [cited 2019 July 22] Available from: http://www . nationalpostdoc .org/?page=PD A_toolkit

[5] Eckardt P, Hammer MJ, Barton-Burke M, et al. All nurses need to be research nurses. Journal of Clinical and Translational Science. 2017; 1(5): 269-270. PMid:29430304 https://doi.org/10.1017/cts . 2017.294

[6] Grady PA, Gough LL. Nursing science: claiming the future. Journal of Nursing Scholarship. 2015; 47(6): 512-521. PMid:26448502 https://doi.org/10.1111/jnu.12170

[7] Ponte PR, Hayman LL, Berry DL, et al. A new model for postdoctoral training: the postdoctoral nursing research fellowship in cancer and health disparities. Nursing Outlook. 2015; 63(2): 189-203. PMid:25771193 https ://doi.org/10.1016/j.outlook. 2014 .11 .014
[8] Tinto V. Dropout from higher education: A theoretical synthesis of recent research. Review of Educational Research. 1975; 45(1): 89-125. https://doi.org/10.3102/00346543045001089

[9] Manson SM. Personal journeys, professional paths: persistence in navigating the crossroads of a research career. Am J Public Health. 2009; 99 Suppl 1: S20-25. PMid:19246673 https://doi.org/10 $.2105 /$ A JPH .2007 .133603

[10] Berwick DM, Nolan TW, Whittington J. The triple aim: care, health, and cost. Health Affairs. 2008; 27(3): 759-769. PMid:18474969 https://doi .org/10.1377/hlthaff .27.3.759

[11] Santucci AK, Lingle JH, Schmidt KL, et al. Peer-mentored research development meeting: a model for successful peer mentoring among junior level researchers. Academy of Psychiatry. 2008; 32(6): 493 497. PMid:19190294 https://doi.org/10.1176/appi.ap.32 .6 .493

[12] Freeman LC. The Development of Social Network Analysis: A Study in the Sociology of Science. Vancouver, BC Canada: Empirical Press; 2004.

[13] Godley J, Barron G, Sharma A. Using social network analysis to assess collaboration in health research. Journal of Healthcare, Science, and the Humanities. 2011; 1: 99-118.

[14] Vacca R, McCarty C, Conlon M, et al. Designing a CTSA-based social network intervention. Clinical and Translational Research. 2015; 8(4): 281-289. PMid:25788258 https ://doi.org/10.1111/cts . 12267

[15] Yeung Y, Timon L, Pun-hon N. A social network analysis of research collaboration in physics education. American Journal of Physics. 2005; 73: 145-150. https ://doi.org/10.1119/1.1775799 